\title{
Interactive comment on "Technical note: A global database of the stable isotopic ratios of meteoric and terrestrial waters" by Annie L. Putman and Gabriel J. Bowen
}

\section{Annie L. Putman and Gabriel J. Bowen}

putmanannie@gmail.com

Received and published: 23 May 2019

The authors would like to thank this reviewer for their kind and constructive comments. Yes, we agree that more description of the different types would be useful for the reader, and will will plan to include a link to the homepage of the wiDB web interface in the document. 